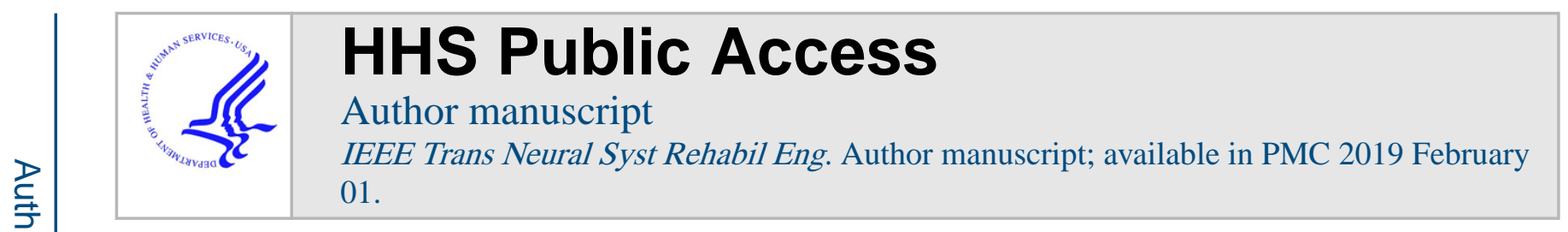

Published in final edited form as:

IEEE Trans Neural Syst Rehabil Eng. 2018 February ; 26(2): 441-450. doi:10.1109/TNSRE.

\title{
$3 \mathrm{~B}^{1} \mathrm{OBb}^{3} \mathrm{e} e \mathrm{ct}$ Recognition of a Robotic Navigation Aid for the \\ Visually Impaired
}

\author{
Cang Ye [Senior Member, IEEE] and Xiangfei Qian
}

\begin{abstract}
This paper presents a 3D object recognition method and its implementation on a Robotic Navigation Aid (RNA) to allow real-time detection of indoor structural objects for the navigation of a blind person. The method segments a point cloud into numerous planar patches and extracts their Inter-Plane Relationships (IPRs). Based on the existing IPRs of the object models, the method defines 6 High Level Features (HLFs) and determines the HLFs for each patch. A Gaussian-Mixture-Model-based plane classifier is then devised to classify each planar patch into one belonging to a particular object model. Finally, a recursive plane clustering procedure is used to cluster the classified planes into the model objects. As the proposed method uses geometric context to detect an object, it is robust to the object's visual appearance change. As a result, it is ideal for detecting structural objects (e.g., stairways, doorways, etc.). In addition, it has high scalability and parallelism. The method is also capable of detecting some indoor non-structural objects. Experimental results demonstrate that the proposed method has a high success rate in object recognition.
\end{abstract}

\section{Index Terms}

Robotic Navigation Aid; Visually Impaired; 3D Object Recognition; Geometric Context; Gaussian Mixture Model

\section{Introduction}

Visual impairment reduces a person's independent mobility and severely deteriorates the quality of life. According to the estimates from the World Health Organization, there are 285 million people with visual impairment, of which 39 million are blind. Because the leading cause of vison loss is age related diseases and the world population is aging rapidly, we will see more people experiencing visual impairment in the coming decades. Therefore, there is a dire need for developing new navigation aids to allow visually impaired persons move around safely and help them live independent lives.

Just like guiding a mobile robot, the blind navigation problem includes obstacle avoidance and wayfinding. Obstacle avoidance is a local problem of taking steps safely without bumping into anything, tripping, or falling, while wayfinding deals with a global problem of planning and following a path towards the destination. Object recognition is a crucial component for both obstacle avoidance and wayfinding. A detected object can be treated as an obstacle to trigger obstacle avoidance or used as a waypoint to lead a blind traveler to the destination. Despite significant advances in computer vision in the past decades, independent 
mobility still poses a great challenge to a visually impaired individual. Up to date, white cane remains the prevailing mobility tool because of its powerful haptic feedback and low cost. However, a white cane can only provide point-contact information instead of a "full picture" of its surroundings and has a limited range that is too nearsighted for path planning. Moreover, it is unable to provide location information for wayfinding. To address these limitations, a number of Robotic Navigation Aids (RNAs) have been introduced for the visually impaired. However, there is currently no RNA that has effectively addressed both obstacle avoidance and wayfinding problems. The main technical challenge is that both problems must be addressed in a small platform with limited resources.

This paper considers independent mobility in indoor environments. An indoor environment presents a greater challenge for navigation than an outdoor one because: (1) it has a higher obstacle density; (2) it may contain overhanging obstacles; and (3) it is GPS-denied. The first two conditions require 3D perception while the third makes it impossible to use existing GPS-based navigation methods [1]. However, an indoor environment may provide the RNA with off-board computing support and allows for a small system with high autonomy. To take this advantage, we developed a portable RNA [2], [3] with a client-server architecture (described in II). The RNA, called Smart Cane (SC), is a computer-vision-enhanced white cane. It provides two functions, wayfinding [4], [5] and 3D object recognition [6], to its user by simultaneously processing the intensity and range data from a 3D camera. The wayfinding function is achieved by using a simultaneous localization and mapping (SLAM) method [7] to locate the user's position. This paper will focus itself on object recognition. An overview of the related work on object detection with RNAs is given in the reminder of this section.

\section{A. Related Work in RNAs with Obstacle/Object Detection}

The existing RNAs can be classified into three categories: Robotic Wheelchair (RW) [8], Robotic Guide Dog (RGD) [9], [10] and Electronic White Cane (EWC) [11], [12], [13], [14]. RW and RGD lack portability and may limit their uses in a single floor space. An EWC is a handheld/wearable device capable of detecting an obstacle in its immediate surrounding. It uses sonar(s) [11], [12] or laser-based triangulation [13], [14] for obstacle detection. In spite of the portability, this kind of devices cannot provide the user with a "full picture" of the surrounding for object recognition owing to the use of a single-point range sensor. In addition, they are unable to provide location information for wayfinding.

\section{B. Related Work in Object Recognition}

Object-level information is critically important for an RNA to navigate the blind user in an indoor environment. Objects like doorways/stairways can be used as waypoints to guide the user to move towards the destination while other objects must be treated as obstacles and the related information is used for obstacle avoidance. Indoor objects can be classified into two types, structural objects and non-structural objects. Structural objects are permanent fixtures built into the structures, while non-structural objects are the other indoor objects whose position can be changed. In this work, we only consider objects consisting of planar surfaces. Our target structural objects include doorway, hallway, stairway, ground and wall. The target non-structural objects include table, monitor and parallelepiped (computer case as 
an embodiment). Most of these objects' visual appearances may change from time to time. For instances, a poster on a door/wall may completely change the visual appearance of the door/wall and a monitor may look very different when it is on and off. This means that an object recognition method solely based on visual information cannot reliably detect indoor objects. On the contrary, 3D geometric features (e.g., size, orientation, height, etc.) of these objects are constant and therefore can be used for reliable object detection.

Object recognition by using visual information is performed in a 2D image space. Visual feature matching [15] and visual shape matching [16] have been employed for single-view object detection. They can also be jointly used to improve object recognition result [17]. Recently, visual codebook [18] and bag of boundaries [19] (mid-level visual feature/shape) were introduced for multi-view object recognition (i.e., concurrent pose and object recognition). With respect to structural object detection, Tian et al. [20] proposed to extract edges and corners of a door frame from an image and match them with that of the geometric door model for door detection. They used the door detection method for indoor wayfinding of a blind traveler. The high visual appearance variability of an indoor environment may pose challenges to reliable extraction of visual/shape features and thus affect object recognition result. Bills et al. [21] proposed to extract line segments and vanishing point from the image of a camera. Their method then took the largest horizontal-line cluster as a stairway and the vanishing point a corridor. However, the method is not reliable as other objects (e.g. a window blind) or parallel-line patterns presented in the environment can also produce a horizontal-line cluster and it is unlikely to extract the vanishing point for a hallway in our case due to the camera's short range and low position.

Object recognition by using geometric features, on the contrary, is done in 3D data (point cloud) space. Existing methods recognize a 3D object by matching key points [22], [23], [24] or Implicit Polynomials (IPs) [25] between two objects. Spin image [22], [24] and shape spectrum descriptor [23] are two typical key points used for 3D object recognition. A key point based method requires a large number of descriptors from multiple viewpoints on the object surface to perform surface matching and thus results in a very high computational cost. An IP matching method is more computationally efficient as IPs are capable of describing complicated object boundaries. However, the runtimes for detecting a 3D object is still over 10 seconds [25]. Moreover, all these methods use an object's local geometric features only and are thus not suitable for detecting some indoor structural objects where the geometric contexts (inter-object geometric relationships) are important attributes.

Xiong and Huber [26] introduced a Conditional Random Field (CRF) model to classify a 3D scene's planar patches by using both local features and geometric context. The method defines four types of object models—-wall, floor, ceiling and clutter (i.e., unclassified object). Each of them is a single-plane-object. It first extracts from each planar patch a number of local features (orientation, area and height) and contextual features (inter-plane relationships) including orthogonal, parallel, adjacent and coplanar. By using these features, the method constructs a graph connecting each planar patch with its $k$-nearest neighbors. Finally, a CRF model taking into account the local and contextual features is used to compute, for each object, the likelihood of being one of the four model objects. Anand et al. [27] employed an undirected graph to model a 3D scene. In the graph, a node (node feature) 
describes the visual appearance and geometric attributes of a segment while an edge (edge feature) describes the inter-segment relationships. They proposed a discriminant function, computed as a weighted sum of the node and edge features, to describe a predicted classification of the scene. The objective is to find a classification that maximizes the discriminant value. Their method uses 48 node features and 11 edge features to recognize up to 9 different objects in an office environment. Both methods incur high computational cost. Taking [27] as an example, it takes $\sim 18$ minutes to identify 17 objects from a scene with 50 segments. The method's complexity for identifying $m$ objects out of a scene of $N$ segments is $O\left(m^{N}\right)$, i.e., NP hard. Apparently, it is unsuitable for the SC application that requires realtime object recognition.

In this paper, we extend our earlier work [6] and introduce a new method for detecting the eight aforementioned objects. The method divides the object recognition problem into four stages. First, it segments a 3D point cloud into planar patches. Second, it extracts the InterPlane Relationships (IPRs) between each two planar patches and classifies the planar patches into 6 categories, each of which has an exclusive High Level Features (HLF). Each HLF is a set of IPRs existing in one object model. Third, a plane classifier is used to classify a planar patch with a particular HLF into a plane belonging to the corresponding object model by using the patch's local attributes and the parameter(s) of the IPR(s). Finally, it clusters a number of planar patches of the same type into a model object in an iterative fashion. In this work, 6 HLFs are defined on top of 9 IPRs existing in the 8 object models. As the method uses only 6 HLFs and discards a tremendous number of unwanted IPR combinations, the computational cost for object recognition is substantially reduced. This overcomes the NP complexity problem of the CRF-based method [26].

\section{Smart Cane Prototype}

The SC is intended to enhance rather than replace a white cane's function. As depicted in Fig. 1, a forward-looking 3D camera is mounted on a white cane to perceive the surroundings and a user-worn mobile computer processes the camera's data into navigational information. The SC degrades itself into a conventional white cane if the computer vision functions fail. In order to achieve necessary SLAM accuracy, a 3D time-offlight camera (SwissRanger SR4000) is used in this work. Compared with a stereo camera, the SR4000 provides more accurate range data. It also provides more complete range data than a stereo or RGB-D camera for object detection. The camera has a $176 \times 144$ pixels resolution and a $43.6^{\circ} \times 34.6^{\circ}$ field of view. Its small dimension $\left(50 \times 48 \times 65 \mathrm{~mm}^{3}\right)$ makes it suitable for the SC. The camera illuminates its environment with modulated infrared light and detects a range up to 5 meters (with $\pm 1 \mathrm{~cm}$ accuracy) based on phase shift. It produces intensity and range data simultaneously at a rate up to $50 \mathrm{fps}$. The camera is tilted up by $18^{\circ}$ to make the cane body outside the camera's field of view.

The camera's intensity and range data are processed to determine the device's pose change between each two camera views [28], based on which a graph SLAM method [7] computes the device's pose in the world coordinate system. The pose information is then used for wayfinding and 3D data registration. For object recognition, the 3D point cloud is segmented 
into planar segments, which are then classified and clustered into the target objects. Finally, the detected objects are used for obstacle avoidance or as navigational waypoints.

The first SC prototype uses a client-server architecture for real-time computing. An HP Stream 7 tablet is used as the client and a desktop computer (i7-3370K CPU, 8GB memory, Windows 7 64-bit) as the server. The client computer accesses the server via WIFI for object recognition service. In this way, the client only needs to perform low-computational tasks. Such a computing arrangement allows the SC to perform real-time object detection and wayfinding. A speech interface is used for human-device interaction. It takes voice commands from the user and announces object recognition results to the user.

Fig. 2 is the diagram of the SC prototype's software. The user speaks to the headset. The Speech Interface Module (SIM) converts the voice command into a file containing the name of the object to be detected and sends the file to the desktop server. The Object Recognition Module (ORM) then acquires data from the SR4000 through the tablet computer by using the SR4K Receiver Module (SRM) and the SR4K Acquisition \& Transmission Module (SATM) and detects the target object. The detection result, a text string containing the object name, is sent to the SIM, which then converts the text into speech and makes announcement to the user via the Bluetooth headset. Handshaking protocol is designed and used to ensure error-free data communication between the SRM and SATM and between the SIM and ORM. To reduce wireless data traffic, the SATM only sends the camera's intensity and depth data to the SRM, which then uses the camera model to convert the depth data into the $x, y, z$ coordinate values for each data point.

\section{Object Recognition Algorithm}

The proposed object recognition method is depicted in Fig. 3. It consists of five main procedures: 3D point cloud data acquisition, plane extraction from point cloud, feature extraction, design and training of Gaussian Mixture Model based Plane Classifier (GMMPC), and plane clustering. Each of them will be described in this section.

\section{A. Range Data Acquisition and Plane Extraction}

3D point data are captured from the SR4000 frame by frame and registered to form a large view of the scene by using the camera poses. The multi-view point cloud data is then segmented into $N$ planar patches by the NCC-RANSAC method [29].

\section{B. Features and Feature Vectors}

A planar patch's properties describing its intrinsic attributes and geometric context are defined as the planar patch's features. In this paper, the geometric context refers to InterPlane Relationships (IPRs). The features of a planar patch are represented by a feature vector, which will be used later to classify the patch into one belonging to a specific type of objects. For each of the $N$ extracted planar patches, a feature vector construction procedure is performed to assign each of them a feature vector. In this paper, we define three classes of features-Basic Feature (BF), Low Level Feature (LLF), and High Level Feature (HLF). 
1) Basic Features-BFs are local features that describe a planar patch's intrinsic attributes. They serve as the identity of the planar patch and play important roles in object recognition. Similar to [26], three BFs—Orientation, Area, and Height (OAH)—are defined for a patch. In this paper, the OAH of the $i^{\text {th }}$ patch are computed by the following three equations:

$$
O_{i}=\operatorname{arcos}\left(\mathbf{n}_{\mathbf{i}} \cdot \boldsymbol{Z}\right),
$$

where $\mathbf{n}_{\mathbf{i}}$ is the patch's normal and $Z=(0,0,1)$ is the unit vector of $Z$-axis;

$$
\gamma A_{i}=\sum_{j=1}^{K}\left(\frac{h}{f}\right)^{2} d_{i j}^{2}=\sum_{j=1}^{K} d_{i j}^{2}
$$

where $d_{i j}$ is the distance from the $j^{\text {th }}$ point of the patch to the camera, $h$ is the pixel width/ pitch of the SR4000, and $f$ is the camera's focal length; and

$$
H_{i}=\left.\max \left(z_{i j}\right)\right|_{j=1, \cdots, K},
$$

where $z_{i j}$ is the $z$ coordinate of the $j^{\text {th }}$ point and $K$ is the total number of points of the $i^{\text {th }}$ patch. (2) is an approximate method for calculating the $i^{\text {th }}$ patch's area. The method uses a pinhole camera model and assumes that an image pixel occupies a square area. While constant $\gamma$ in (2) may be determined using the camera's parameter, its actual value does not affect the proposed object recognition method as long as the same value is used in both the training and classification phases. For simplicity, we use $\gamma=1$.

2) Low Level Features-To classify a planar patch into a constituent element of a model object, the patch's IPRs must be considered in addition to its BFs. In this paper, the following 9 IPRs are defined for patch $P_{i}$ with reference to patch $P_{j}$ :

plane-distance is a value representing the minimum distance between the points of $P_{i}$ and the points of $P_{j}$.

plane-angle represents the angle between $P_{i}$ and $P_{j}$. It is computed as $a_{i j}=\operatorname{arcos}\left(\mathbf{n}_{\mathbf{i}}, \cdot \mathbf{n}_{\mathbf{j}}\right)$

parallel-distance is the distance between two parallel planes, $P_{i}$ and $P_{\mathrm{j}}$. This distance, denoted $d_{i j}^{\prime \prime}$ is computed as the mean of the distance from the centroid of $P_{i}$ to patch $P_{j}$ and the distance from the centroid of $P_{j}$ to patch $P_{i}$.

projection-overlap-rate is a value representing to what extent $P_{i}$ overlaps $P_{j}$.

projection-distance is the minimum distance from the points of $P_{i}$ to patch $P_{j}$. 
Fig. 4 illustrates the definitions of the above LLFs. The definitions for plane-distance, planeangle and parallel-distance are self-explanatory. Readers are referred to [27] for details. Fig. $4 \mathrm{c}$ illustrates how the two new LLFs, projection-overlaprate and projection-distance for plane $P_{i}$ with reference to $P_{j}$, are calculated. For simplicity, we use a $2 \mathrm{D}$ illustration. $L_{j}$ represents the size of patch $P_{j}$ and $L_{i j}$ stands for the size of the overlap region. The projection-overlap-rate of $P_{i}$ with reference to $P_{j}$ is $L_{i j} / L_{j}$ and the projection-distance of $P_{i}$ with reference to $P_{j}$ is $d_{i j}$.

is-parallel describes if $P_{i}$ is parallel to $P_{j}$. A value of 1 means that $P_{i}$ is parallel to $P_{j}$; while a value of 0 means they are not parallel. Detection of this relationship within the SR4000's data requires taking into account the camera's noise. For this reason, we let is-parallel $=1$ if $\mid$ $\mathrm{a}_{i j} j<5^{\circ}$ and $l_{i}^{j} \geq 5 \mathrm{~cm}$, or is-paralle $=0$ otherwise.

is-perpendicular describes if $P_{i}$ is perpendicular to $P_{j}$. To account for the sensor's noise, we let is-perpendicular $=1$ if $\left|\mathrm{a}_{i j}-90^{\circ}\right|<5^{\circ}$, or is-perpendicular $=0$ otherwise.

is-coplanar describes if $P_{i}$ is co-planar with $P_{j}$. iscoplanar $=1$ if $\left|\mathrm{a}_{i j}\right|<5^{\circ}$ and $d_{i j}^{\prime \prime}<5 \mathrm{~cm}$, otherwise iscoplanar $=0$.

is-adjacent describe if $P_{i}$ is adjacent to $P_{j}$. is-adjacent 1 if the plane-distance between $P_{i}$ and $P_{j}$ is smaller than $7 \mathrm{~cm}$, otherwise $i s$-adjacent $=0$.

Since there are $N$ planar patches, an $N \times N$ matrix (using bold-face denotation) is formed to record each of the 9 IPRs among the $N$ patches. Each matrix is called an LLF, which is denoted by the corresponding IPR in boldface. For instance, is-parallel stands for the isparallel matrix. In an LLF matrix, an element at $(i, j)$ (i.e., row $i$ and column $j$ ) describes the IPR of $P_{i}$ with reference to $P_{j}$. For example, is-parallel $(i, j)=1$ indicates that $P_{i}$ is parallel to $P_{j}$.

3) High Level Features and Feature Vectors-The goal of object recognition is to identify one of the eight objects, namely, doorway, hallway, stairway, parallelepiped, monitor, table, ground and wall, from a scene. In this work, each planar patch is classified into a plane belonging to one of the eight objects (models). The LLFs cannot be directly used to perform such a classification task. To address this, we define 6 new High Level Features (HLFs) as shown in Fig. 5. These features are mutually exclusive. Each HLF represents a set of particular IPRs that exists in a particular model. The HLF extraction is a process to identify the HLF for each of the $N$ planar patches and assign each patch a HLF vector. The $\mathrm{BFs}(\mathrm{OAH})$ extracted earlier from each patch are used in constructing the HLF vector. We first construct a $\mathrm{BF}$ vector $[O, A, H]$ for each planar patch. Each $\mathrm{BF}$ vector is then extended based on the patch's HLF. For a planar patch with HLF-1, HLF-2, HLF-3 or HLF-6, we add parameter $d$ into its BF vector to form a HLF vector $[O, A, H, d]$. For a patch with HLF-5, we add parameter $d_{1}$ and $d_{2}$ to form a HLF vector $\left[O, A, H, d_{1}, d_{2}\right]$. For a patch with HLF-4, we simply use the BF vector as the HLF vector. In this work, a plane is treated as an object if it is a wall/ground. Such a plane does not have an IPR. We therefore simply use its BF vector as the HLF vector. A plane with an HLF as depicted in Fig. 5 is called a 
complex plane, while a wall/ground plane is called an elementary plane. An elementary plane is a standalone one without an IPR.

The HLF detection and HLF vector assignment for the $N$ planar patches are achieved by analyzing the LLF matrices according to the following procedures:

HLF-1 (coplanar with distance $d$, Fig. 5a): examine iscoplanar and find all elements with value " 1 "; for each element $e_{i j}=1$, find the corresponding $d$ value from planedistance; form HLF-1 vector $[O, A, H, d]$ and assign it to the planar patch.

HLF-2 (parallel and face-to-face with distance $d$, Fig. 5b): examine is-parallel and find all elements with value "1"; for each element $e_{i j}=1$, ensure the two planes are face-to-face by checking their normals; examine projection-overlap-rate and ensure the elements at $(i, j)$ and $(j, i)$ are larger than 0.7 ; let $d=$ parallel-distance $(i, j)$; and form HLF-2 vector $[O, A, H, d]$ and assign it to patch $P_{i}$.

HLF-3 (step-shape with distance $d$, Fig. 5c): examine isparallel and find all elements with value "1"; for each element $e_{i j}=1$, examine projection-overlap-rate and ensure the elements at $(i, j)$ and $(j, i)$ are 0 ; let $d=$ parallel-distance $(i, j)$; form HLF-3 vector $[O, A, H, d]$ and assign it to patch $P_{i}$.

HLF-4 (parallelepiped-shape, Fig. 5d): examine is-perpendicular and find all elements with value " 1 "; for each element $e_{i j}=1$, ensure is-adjacent $(i, j)=1$; perform the same procedure for each of $e_{i k}$ and $e_{j k}$, and ensure perpendicular and adjacent relations; confirm the corner point's convexity; form HLF-4 vector $[O, A, H]$ and assign it to patch $P_{i}$. To determine the corner point's convexity, we calculate the centroid of the data points of the three planes. If its $Y$ coordinate is bigger than that of the corner point, the convexity is asserted.

HLF-5 (side-above with distance $d_{1}$ and $d_{2}$, Fig. 5e): examine plane-angle and find all elements with a value in $\left[90^{\circ}, 130^{\circ}\right]$; for each of these elements $e_{i j}$, let $d_{1}=$ projectiondistance $(i, j)$ and compute $d_{2}$; form HLF-5 vector $\left[O, A, H, d_{1}, d_{2}\right]$ and assign it to patch $P_{i}$.

HLF-6 (above with distance $d$, Fig. 5f): examine is-parallel and find all elements with value "1"; for each element $e_{i j}=1$, ensure that the two planes face the same direction by checking their normals; ensure projection-overlap-rate $(i, j)=1.0$; let $d=$ paralleldistance $(i, j)$; form HLF-6 vector $[O, A, H, d]$ and assign it to patch $P_{i}$.

The HLF assignment process also generates 6 matrices, $Q_{1}, \ldots, Q_{6}$, each of which records the $N$ planar patches' IPRs for HLF-1, .., HLF-6, respectively. The HLF extractor in essence serves as an IPR classifier that identifies if a given planar patch has a geometric context as defined in Fig. 5. After HLF vector assignment, the HLF vectors are sent to the GMM GMM-PC (Fig. 3) for plane classification. It is noted that the complexity for identifying a plane with HLF-1, HLF-2, HLF-3, HLF-5 or HLF-6 among $N$ planar patches is $O(N)$ and that of HLF-4 is $O\left(N^{2}\right)$. This means that the complexity for classification of a plane to be one belonging to a doorway, hallway, stairway, monitor or object-on-a-table is $O(N)$ and that of one belonging to a parallelepiped is $O\left(N^{2}\right)$. The HLF definition results in real-time plane classification. 
C. GMM-PC-The GMM-PC consists of 8 GMMs, each of which has been trained using data captured from a particular type of objects and is thus able to identify a plane related to that type of object in the presence of the relevant HLF vector. For instance, a planar patch with a HLF-1 vector is classified as a doorway-plane (i.e., a plane belonging to a doorway) if the parameters of the vector $[O, A, H, d]$ are right. This is because the HLF-1 vector causes the doorway-GMM to produce a large response-a likelihood value for the plane to be a doorway-plane. In this work, the GMMs are trained using training data in such a way that planar patch A with HLF-1, HLF-2, ..., and HLF-6 is detected as a plane of a doorway, hallway, stairway, parallelepiped, monitor, and object-on-a-table, respectively.

In this subsection, we present the technical details and training procedure of the GMMs, as well as plane classification by the GMM-PC. For simplicity, we call a GMM for detecting object type $O_{k}$, for $k=1, \cdots, 8$, an $O_{k}$-GMM. Here, $O_{1}, O_{2}, \cdots, O_{8}$ represent doorway, hallway, stairway, parallelepiped, monitor, table, ground and wall, respectively. We also call a plane belonging to $O_{k}$ an $O_{k}$ plane and a scene with object $O_{k}$ an $O_{k}$ scene. In the GMMPC, the $O_{i^{-}}$GMM receives all HLF- $i$ vectors and identify if the associated patch is an $O_{i^{-}}$ plane (here, $i=1, \cdots, 6$ ). The ground-GMM and the wall-GMM receive the remaining BFs and classify each of the associated planar patches into a ground-plane or a wall-plane.

1) Gaussian Mixture Model-A GMM [30] is a probability density function represented as a weighted sum of $M$ Gaussian component densities. It is given by:

$$
p(\boldsymbol{x} \mid \lambda)=\sum_{i=1}^{M} \omega_{i} g\left(\boldsymbol{x} \mid \boldsymbol{\mu}_{i}, \sum_{i}\right),
$$

where $x$ is a $D$-dimensional (in our case, $D=3,4$ or 5 ) vector with continuous values, $\omega_{\mathrm{i}}, \mathrm{i}=$ $1, \ldots, \mathrm{M}$, are the mixture weights, and $g\left(x \mid \boldsymbol{\mu}_{i}, \Sigma_{i}\right), i=1, \ldots, M$, are the component Gaussian densities. Each component density is a $D$-variate Gaussian function given by:

$$
g\left(\boldsymbol{x} \mid \boldsymbol{\mu}_{i}, \sum_{i}\right)=\frac{\exp \left\{-\frac{1}{2}\left(\boldsymbol{x}-\boldsymbol{\mu}_{\boldsymbol{i}}\right)^{\prime} \sum_{\boldsymbol{i}}^{-\mathbf{1}}\left(\boldsymbol{x}-\boldsymbol{\mu}_{\boldsymbol{i}}\right)\right\}}{(2 \pi)^{D / 2}\left|\sum_{i}\right|^{1 / 2}}
$$

where $\mu_{i}$ and $\Sigma_{i}$ are the mean vector and covariance matrix, respectively. The mixture weights satisfy $\sum_{i=1}^{M} \omega_{i}=1$. The complete GMM is parameterized by $\boldsymbol{\mu}_{i}, \Sigma_{i}$ and $\omega_{i}$ for $i=1$, ...,M. We denote these parameters collectively by $\lambda$ from now on for conciseness.

In our case, the configuration ( $M$ and $\lambda$ ) of an $O_{i}$-GMM is estimated by training the model using a set of HLF vectors obtained from scenes with $O_{k}$ type of objects. For example, a stairway-GMM is trained by a set of HLF-3 vectors extracted from various stairway-scenes. A GMM training process is to estimate the model's configuration such that the GMM best matches the distribution of the HLF vectors for a particular type of objects. The most popular and well-established method for estimating the parameters of a GMM is the Maximum Likelihood (ML) estimation [31]. The objective of ML estimation is to find the model parameters that maximize the likelihood of the GMM given the training data. For a 
sequence of $T$ independent training vectors, $X=\left\{x_{1}, x_{2}, \ldots, x_{T}\right.$, the GMM likelihood is given by,

$$
p(\boldsymbol{X} \mid \lambda)=\prod_{t=1}^{T} p\left(\boldsymbol{x}_{\boldsymbol{t}} \mid \lambda\right)
$$

Since this expression is a non-linear function of $\lambda$, a direct maximization of this expression is not possible. However, ML parameter estimates can be obtained iteratively by the Expectation-Maximization (EM) algorithm [31].

The EM algorithm begins with an initial model $\lambda$ (a set of random values) and estimates a new model $\bar{\lambda}$, such that $p(X \mid \bar{\lambda}) \geq p(X \mid \lambda)$. The new model is taken as the initial model for the next iteration and the process is repeated until a convergence is achieved. The details on computing $\bar{\lambda}$ (i.e., $\overline{\omega_{i}}, \overline{\mu_{i}}$ and $\bar{\sigma}_{i}^{2}$ ) are referred to [30]. Each EM iteration guarantees a monotonic increase in the model's likelihood value.

After the EM process, the posteriori probability for component $i$ of the GMM is given by

$$
\operatorname{Pr}\left(i \mid \boldsymbol{x}_{\boldsymbol{t}}, \lambda\right)=\frac{\omega_{i} g\left(\boldsymbol{x}_{\boldsymbol{t}} \mid \boldsymbol{\mu}_{\boldsymbol{i}}, \sum_{i}\right)}{\sum_{k=1}^{M} \omega_{k} g\left(\boldsymbol{x}_{\boldsymbol{t}} \mid \boldsymbol{\mu}_{\boldsymbol{k}}, \sum_{\boldsymbol{k}}\right)},
$$

In this work, the number of the component Gaussian densities, $M$, is determined by repeating the training process with an increasing $i$ and observing the trained GMM's output $(p(x \mid \lambda))$. If the mean of the output difference between an $I$-component GMM and an $(I+1)$ component GMM,

$$
\gamma \omega=\frac{\sum_{t=1}^{T}\left(\sum_{i=1}^{I+1^{\prime}} g\left(\boldsymbol{x}_{t} \mid \boldsymbol{\mu}_{\boldsymbol{i}}^{\prime}, \sum_{i}^{\prime}\right)-\sum_{i=1}^{I} \omega_{i} g\left(\boldsymbol{x}_{t} \mid \boldsymbol{\mu}_{i}, \sum_{i}\right)\right)}{T},
$$

is below a threshold, we let $M=I$ because more Gaussian component densities in (4) will not change the probability density of the GMM. In other words, the GMM with configuration $M=I$ and $\lambda$ found by the EM algorithm is sufficient to describe the distribution of the training vectors.

2) Training of GMMs-We used a Pan-Tilt Unit (PTU) to acquire 3D point data. The ground truth pose of the PTU were used for data registration. To train $O_{k} \mathrm{GMM}(k=1, \cdots$, 8 ), we obtained 500 point cloud datasets from various $O_{k}$-scenes $(k=1, \cdots, 8)$ in 4 buildings on campus. Through plane segmentation and feature extraction, we obtained a set of HLF vectors from each of the datasets. We then trained $O_{k}$-GMM using the HLF vectors of all datasets. The training process determined the configuration ( $M$ and $\lambda$ ) of $O_{k}$-GMM. 
The smallest probability density $p_{m i n}^{k}$ given by the $O_{k}$-GMM for the training data is recorded as the threshold for plane classification in a later stage.

3) Plane Classification by GMMs-Once trained, each GMM in the GMM-PC is able to classify a planar patch into one of the eight plane types using the patch's HLF vector. To be specific, a planar patch's HLF (or BF if HLF is unavailable) vector is presented to the relevant GMM. The GMM's output, $p_{k}(\mathbf{X} \mid \lambda)$ for $k=1, \cdots, 8$ is compared with the early recorded threshold $p_{\text {min }}^{k}$. If $p_{k}(\boldsymbol{X} \mid \lambda)>p_{\text {min }}^{k}$, the planar patch is classified as an $O_{k}$-plane. Based on the HLF vectors we define, a planar patch captured from a wall/ground may be classified as both an elementary plane and a complex one. For instance, a patch from a ground may be classified as a ground-plane and a stairway-plane. In this case, the complex plane classification overrides the elementary plane classification. All extracted HLF vectors are then presented to the GMM-PC and the corresponding planar patches are classified into the eight types of planes. The GMM-PC results in 8 arrays, $G_{k}$ for $k=1, \cdots, 8$, each of which stores the indexes of the planar patches that have been classified as $O_{k}$-planes.

4) Recursive Plane Clustering-In this stage, the classified planes, $O_{k}$-planes for $k=1$, . $\cdots, 4$, are recursively clustered into a number of objects, $O_{k}$ for $k=1, \cdots, 4$. This means that the doorway-/hallway-/stairway-/parallelepiped-planes are grouped into doorway(s)/ hallway(s)/stairway(s)/parallelepiped(s) by a Recursive Plane Clustering (RPC) process. A monitor-/table-/ground-/wall-plane is treated as a single-plane object and thus no further process is needed. Four independent procedures, RPC-1, RPC-2, RPC-3 and RPC-4, process $G_{1}, G_{2}, G_{3}$ and $G_{4}$, respectively and cluster the object-planes into the four types of objects. For conciseness, the stairway-plane and parallelepiped-plane clustering (RPC-3 and RPC-4) (see Alg. 1) are briefly described while the doorway-plane and hallway-plane clustering (RPC-1 and RPC-2) are omitted due to their simplicities.

\section{Algorithm 1}

Pseudo code of RPC-3/RPC-4: $U_{n}$ is a static array. $n=3$ for RPC- 3 and $n=4$ for RPC-4, respectively.

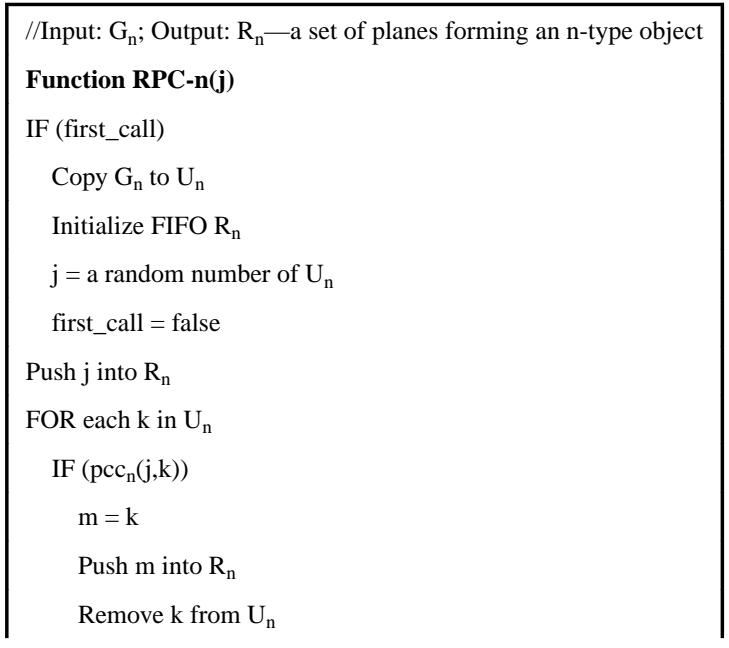


Return RPC_n(m)

Return $\mathrm{R}_{\mathrm{n}}$

Stairway plane clustering (RPC-3): It starts by randomly fetching a number $j$ from $U_{3}$ (a copy of $G_{3}$ ) and pushing it into a First-In-First-Out buffer $R_{3}$. The RPC then pushes each of the remaining number $k$ of $U_{3}$ into $R_{3}$ by calling itself recursively if $p c_{3}(j, k)=\left(Q_{3}(j, k) \|\right.$ (is-perpendicular $(j, k) \& \&$ is-adjacent $(j, k)$ ) is 1 . The recursion stops if $\operatorname{pcc}_{3}(j, k)$ is 0 , i.e., no more plane of the same stairway can be found. The planar patches indexed by the numbers in $R_{3}$ are then clustered as a stairway object. After removing these numbers from $G_{3}$ and clearing $R_{3}$, RPC-3 is repeated to cluster the next stairway until $G_{3}$ is empty.

Parallelepiped plane clustering (RPC-4): RPC-4 operates in the same way as RPC-3 except that $\operatorname{pcc}_{4}(j, k)=Q_{4}(j, k)$ is used in the IF statement.

\section{Experimental Results}

After the eight GMMs were trained, we tested the object recognition method by experiments. We collected another 60 data sets from each type of scenes, half (type I) from the same scenes used for training data collection and the other half (type II) from similar scenes with the same type of objects. We ran the proposed method on the 480 (8.60) datasets and evaluated its performance in term of the success rate of object recognition and runtime.

\section{A. Success Rate of Object Recognition}

Figures 6, 7 and 8 depict the object recognition results of a stairway, doorway, hallway, monitor, and parallelepiped. In all cases, the proposed method detects the target objects correctly. Taking the stairway scene (Fig. 6) as an example, 51 planar patches were extracted. This means that 23000 IPR combinations would need to be processed if the method in [26] or [27] were used. The proposed method extracts only 200 useful HLFs from the planar patches for the subsequent object recognition process and therefore greatly reduced the computational cost. These HLFs are sent to the stairway-GMM for plane classification. Planar patches with large GMM responses (above the threshold) are classified as stairway-planes, which are then grouped into a stairway (Fig. 6c) by the RPC-3 process. Table I shows the proposed method's success rate in object recognition. It can be seen that the average success rate for each type of objects is over $90 \%$ except for monitor. The overall success rate (average rate for all objects) of the proposed method is higher than that of the spin image approach [22] (60\%), the CRF method [26] (90\% for singleplane objects) and the method in [27] (87.9\% for office scenes). For each object type, the success rates of type I data are slightly higher than that of type II data because type I data was acquired from the same scenes used for GMM training data collection. The $93.3 \%$ success rate for type II data indicates that the trained GMMs generalize well. Taking the stairway scenario as an example, the method achieves a $96.7 \%$ success rate for type I data and a $90 \%$ success rate for type II data. In average, its success rate in stairway recognition is $93.3 \%$.

We also compared the GMM method with the Support Vector Machines (SVM) method in plane classification by experiments. The SVM plane classifiers use the SVM library [32] 
with a Gaussian radial basis function kernel and the SVM parameters were determined by the cross-validation and gridsearch method [33]. The result is tabulated in Table I. It can be seen that the GMM method has a slightly better result than the SVM method. SVM is a state-of-the-art classification method that has been widely used in pattern recognition. In practice, its performance can be affected by the selection of SVM kernel and parameters. The need of negative training data (data from scenes with objects other than the eight target objects) also adds difficulty to the training of the plane classifiers. The use of GMM avoids these problems. We ran each GMM-based object detector on the datasets of the other 7 types of objects to obtain its false positive rate. The result indicates a very good object recognition performance: the false positive rates of the detectors for the eight types of objects in Table I are $4.1 \%, 1.4 \%, 3.6 \%, 5.2 \%, 0.7 \%, 6.4 \%, 2.6 \%, 1.2 \%$, respectively. B. Experiments with Multi-View Data In a real-world scenario, the SC's point cloud data may contain multiple views registered by using the estimated device poses. The estimated poses may introduce error to the point cloud data. The object recognition method must S2). The path contains 4 doorways (D1-4), 4 hallways (H1-4) and 2 stairways (S1-2). The object detection ordering is D1, H1, D2, D3, H2, H3, D4, H4, S1 (downwards) and S2 (upwards). The SC was set to its talking-mode to made announcement on the detected object every 1 second along the path. The subject was blind-folded for the test. He/she was accompanied by a research staff for safety and to ensure that the route was followed. The research staff also recorded the experimental results.

accommodate the error. To test this, we collected 3D data from a stairway scene (Fig. 9a) by walking in the environments with the SC. We employed the SLAM method [7] to determine the SC poses and used the poses for data registration. The resulting point cloud (Fig. 9b) were then segmented by the NCC-RANSAC method. The extracted planar segments were classified into various object-planes and eventually grouped into objects labeled in different colors. The object recognition results are shown in Figs. 9c. From the results, we can observe that the stairways (upwards and downwards) and the ground are correctly detected, indicating that the object recognition method is robust to the pose errors.

We also collected a data set from a laboratory environment with 4 computers and 3 tables. In this case, the SC is lifted so that the SR4000 could see the surfaces of the tables and monitors. The proposed method correctly identifies all 3 tables, the monitors of the 4 computers, the walls and the ground. We also carried out tests in other type of environments in several buildings on campus and the results were similar. For conciseness, we omit the results in this paper. These object recognition results demonstrate that the proposed method works well in real wayfinding scenarios. It is noted that the camera data were collected first and processed offline later in these experiments.

\section{B. Human Subject Study}

We recruited seven human subjects from our university to perform experiments with the SC prototype. We followed the protocol approved by the university's institutional review board for recruitment and experiments. The experiments were carried out on the $4^{\text {th }}$ and $5^{\text {th }}$ floor of the ETAS building. Fig. 10 shows the floorplan of the $5^{\text {th }}$ floor. Each subject was required to walk along a predetermined route on the $5^{\text {th }}$ and $4^{\text {th }}$ floors. The route is from a to $\mathrm{c}$ on the 
$5^{\text {th }}$ floor, then from $\mathrm{c}$ to $\mathrm{b}$ (by walking down stairways $\mathrm{S} 1$ and $\mathrm{S} 2$ and stopping at $\mathrm{b}$ on the $4^{\text {th }}$ floor), and finally returns to c (by walking up S1 and S2). The path contains 4 doorways (D1-4), 4 hallways (H1-4) and 2 stairways (S1-2). The object detection ordering is D1, H1, D2, D3, H2, H3, D4, H4, S1 (downwards) and S2 (upwards). The SC was set to its talkingmode to made announcement on the detected object every 1 second along the path. The subject was blind-folded for the test. He/she was accompanied by a research staff for safety and to ensure that the route was followed. The research staff also recorded the experimental results.

Each subject was given a tutorial on how to use the SC before the test. To start the object recognition experiment, the subject spoke "object recognition" to the SC and then "yes" to confirm when prompted by the speech interface. He/she repeated each experiment 4 times. The experimental results (averaged over 4 experiments) for each of the subjects are tabulated in Table II. The average success rates in detecting a doorway, hallway and stairway are $93.8 \%, 96.5 \%$ and $94.7 \%$, respectively, indicating a satisfactory performance in detecting structural objects. It is noted that grounds and walls were always detected by the SC in these experiments. The results are not shown for simplicity. From Table II, we can see that the detection rate of $\mathrm{S} 1$ is much higher than that of $\mathrm{S} 2$. A closer look into the misdetection cases for S2 finds that the camera data are incomplete because the human subject held the SC's handgrip at a lower position in these cases (i.e., the camera's tilt-down angle was smaller). The reflection of the glossy stairway surface caused noticeable missing data that affected plane extraction and thus object recognition. This problem did not occur with S1 though. When the subject was approaching S1, the stairway's treads were not seen by the camera due to their low elevations, resulting in no range data on the upper image plane. This was detected and the system alerted the human subject to raise the handgrip position. By so doing, the camera's tilt down angle was increased to generate a more complete point cloud of the scene.

We carried out more experiments in a number of buildings on campus. The results are similar. For conciseness, only two of them are given here. The first experiment was on the $4^{\text {th }}$ and $5^{\text {th }}$ floors of the ETAS building, containing 6 doorways and 4 hallways on the $5^{\text {th }}$ floor, 10 doorways and 8 hallways on the $4^{\text {th }}$ floor, and 2 stairways in between. The average success rates for doorway, hallway and stairway are $92.2 \%, 92.9 \%$ and 92.9 , respectively. The second experiment was conducted in the EIT building, containing 5 doorways, 9 hallways and 2 stairways. The success rates are $94.3 \%, 94.1 \%$ and $91.2 \%$, respectively. The overall success rates for the three experiments are $93.4 \%, 94.5 \%$ and $92.9 \%$, respectively.

The object recognition method is currently in Matlab codes. It takes the SC about 0.9 second to process one frame of camera data. Because of this, the SC's object detection update rate is $1 \mathrm{~Hz}$ in the experiments. This might cause failure in doorway/hallway detection when the human subject scanned the SC with a fast speed. In this case, the camera only saw a partial doorway/hallway (i.e., one of the walls and the opening space). Therefore, a wall and a ground were detected from the scene instead of a doorway/hallway. Our future plan is to convert the Matlab codes into $\mathrm{C}++$ codes and integrate the pose estimation method with the $\mathrm{SC}$ so that data registration can be performed to avoid the misdetection problem. 
We surveyed the subjects after the experiments. The average ratings for the SC idea, usefulness of object recognition function, usefulness of speech interface, functional enhancement of white cane and comfortability of weight are 4.3, 3.6, 4.4, 4 and 2.7, respectively (5-highest, 1-lowest). The relatively low score for object detection was largely due to the sluggish object detection speed and misdetections. There was a major complain on the weight of the SC (resulting in a low score of 2.7), which caused discomfort when a subject did the four experiments without a break. The survey results suggest the directions for future improvement in the future. It is noted that all subjects reported that object detection helped them take appropriate moves and reduced the need of staff-supervision in route-following. For instances, they reached out for the railing when a stairway was detected and moved straight ahead when a hallway was detected. This means that the environment awareness, as a result of the object recognition function, did help the subjects move around in the environments.

\section{Discussions and Conclusions}

We have presented a method for 3D object recognition in indoor environments for an RNA. The method segments a 3D point cloud into planar patches and classifies each of them into a plane of a model object. It then clusters the classified planes into the model objects. To achieve real-time recognition, we define 9 IPRs, based on which 6 HLFs are defined and used for plane classification. The scheme results in a small number of needed IPRs for plane classification and thus substantially reduces the computational cost in object recognition. Experimental results with real-world data validate the efficacy of the proposed method in term of success rate and runtime. As the method uses one GMM to identify a particular type of object-planes, it has good scalability and parallelism.

To evaluate the method in a real-world navigational setting, we build an RNA prototype and conducted human subject study with the prototype. The results demonstrate the method's efficacy in detecting structural objects and the usefulness of object detection for navigation. It is noted that non-structural objects (such as tables, computer monitors, etc.) detected by the device can provide the user with environment awareness (e.g. in an office/lab) that can be useful for navigation.

In term of future work, we will reduce the device's weight by using a lighter SR4000 or an RGB-D camera. We will also integrate a wayfinding software with the object recognition code to form a complete navigation system. The wayfidning software locates the RNA in a floorplan by a graph SLAM method []. We anticipate that object detection success rate will be improved if data registration is performed. Also, more comprehensive wayfinding functions that use detected stairways/doorways as navigation waypoints will be developed.

\section{References}

1. Balachandran W, Cecelja F, Ptasinski P. A GPS Based Navigation Aid for the Blind. Proc International Conference on Applied Electromagnetics and Communications. 2003:34-36.

2. Ye C. Navigating a Portable Robotic Device by a 3D imaging sensor. Proc IEEE Sensors Conference. 2010:1005-1010. 
3. Ye C, Hong S, Qian X, Wu Wei. Co-Robotic Cane: A New Robotic Navigation Aid for the Visually Impaired. IEEE System, Man, and Cybernetics Magazine. 2016; 2(2):33-42.

4. Ye C, Hong S, Tamjidi A. 6-DOF Pose Estimation of a Robotic Navigation Aid by Tracking Visual and Geometric Features. IEEE Trans Autom Sci Eng. 2015; 12(4):1169-1180. [PubMed: 26924949]

5. Zhang H, Ye C. An Indoor Wayfinding System based on Geometric Features Aided Graph SLAM for the Visually Impaired. IEEE Trans Neural Syst Rehabil Eng. 2017:99.

6. Qian X, Ye C. 3D Object Recognition by Geometric Context and Gaussian-Mixture-Model-Based Plane Classification. Proc IEEE ICRA. 2014:3910-3915.

7. Hong S, Ye C. A Pose Graph based Visual SLAM Algorithm for Robot Pose Estimation. Proc World Automation Congress. 2014:917-922.

8. Levine SP, et al. The Navchair Assistive Wheelchair Navigation System. IEEE Trans Rehabil Eng. 1999; 7(4):443-451. [PubMed: 10609632]

9. Ulrich I, Borenstein J. The GuideCane-Applying Mobile Robot Technologies to Assist the Visually Impaired. IEEE Trans Syst, Man, and Cybern, Part A: Syst and Humans. 2001; 31(2):131-136.

10. Kulyukin V, Gharpure C, Nicholson J, Osborne G. Robot-Assisted Wayfinding for the Visually Impaired in Structured Indoor Environments. Autonomous Robots. 2006; 21(1):29-41.

11. UltraCane. Dec 14. 2012 [Online]. Available: http://www.ultracane.com

12. Bhatlawande S, et al. Design, Development, and Clinical Evaluation of the Electronic Mobility Cane for Vision Rehabilitation. IEEE Trans Neural Syst Rehabil Eng. 2014; 22(6):1148-1158. [PubMed: 24860035]

13. Benjamin JM, Ali NA, Schepis AF. A Laser Cane for the Blind. Proc San Diego Medical Symposium. 1973

14. Yuan D, Manduchi R. A Tool for Range Sensing and Environment Discovery for the Blind. Proc IEEE CVPR Workshops. 2004

15. Lowe D. Object Recognition from Local Scale-Invariant Features. Proc IEEE ICCV. 1999:11501157.

16. Belongie S, Malik J, Puzicha J. Shape Matching and Object Recognition using Shape Contexts. IEEE Trans Pattern Anal Mach Intell. 2002; 24(4):509-522.

17. Lee S, Kim E, Park Y. 3D Object Recognition using Multiple Features for Robotic Manipulation. Proc IEEE ICRA. 2006:3768-3774.

18. Liebelt J, Schmid C, Schertler K. Viewpoint-Independent Object Class Detection using 3D Feature Maps. Proc IEEE CVPR. 2008:1-8.

19. Payet N, Todorovic S. From Contours to 3D Object Detection and Pose Estimation. Proc IEEE ICCV. 2011:983-990.

20. Tian Y, et al. Toward a Computer Vision-based Wayfinding Aid for Blind Persons to Access Unfamiliar Indoor Environments. Machine Vision and Applications. 2013; 24(3):521-535. [PubMed: 23630409]

21. Bills C, et al. Autonomous MAV Flight in Indoor Environments using Single Image Perspective Cues. Proc IEEE ICRA. 2011:5776-5783.

22. Johnson A, Hebert M. Using Spin Images for Efficient Object Recognition in Cluttered 3D Scenes. IEEE Trans Pattern Anal Mach Intell. 1999; 21(5):433-449.

23. Dorai C, Jain AK. Shape Spectrum Based View Grouping and Matching of 3D Free-Form Objects. IEEE Trans Pattern Anal Mach Intell. 1997; 19(10):1139-1145.

24. Lai K, Dieter F. Object recognition in 3D point clouds using web data and domain adaptation. The International Journal of Robotics Research. 2010; 29(8):1019-1037.

25. Ben-Yaacov H, Malah D, Barzohar M. Recognition of 3D Objects Based on Implicit Polynomials. IEEE Trans Pattern Anal Mach Intell. 2010; 32(5):954-960. [PubMed: 20299718]

26. Xiong X, Huber D. Using Context to Create Sematic 3D Models of Indoor Environments. Proc British Machine Vision Conference. 2010:1-11.

27. Anand A, et al. Contextually Guided Semantic Labeling and Search for Three-Dimensional Point Clouds. The International Journal of Robotics Research. 2012; 32(1):19-34.

28. Ye C, Bruch M. A Visual Odometry Method based on the SwissRanger SR-4000. Proc SPIE Defense, Security, and Sensing Symposium. 2010 
29. Qian X, Ye C. NCC-RANSAC: A Fast Plane Extraction Method for Navigating a Smart Cane for the Visually Impaired. Proc IEEE Int Conf Automation Science and Engineering. 2013:267-273.

30. Reynolds, D. Encyclopedia of Biometric Recognition. Springer; Feb. 2008 Gaussian Mixture Models.

31. Dempster AP, Laird NM, Rubin DB. Maximum Likelihood from Incomplete Data via the EM Algorithm. Journal of the Royal Statistical Society, Series B. 1977; 39(1):1-38.

\section{Biographies}

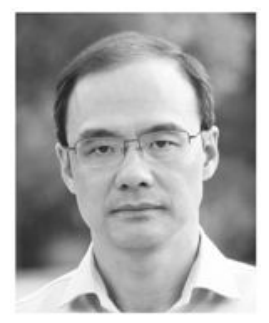

Cang Ye (S'97-M'00-SM'05) received the B. E. and M. E. degrees from the University of Science and Technology of China, Hefei, Anhui, in 1988 and 1991, respectively, and the Ph.D. degree from the University of Hong Kong, Hong Kong in 1999. He is currently a Professor with the Department of Computer Science, Virginia Commonwealth University. His research interests are in mobile robotics, intelligent system, and computer vision.

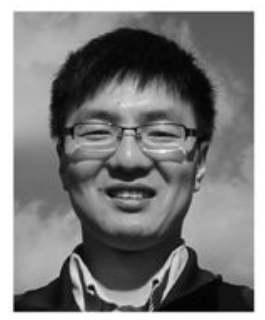

Xiangfei Qian (SM'14) received BS degrees in Computer Science from Nanjing University, Nanjing, China, in 2011, and Ph.D. degree from the University of Arkansas at Little Rock in 2016. He is currently a research engineer at HP Headquarters, Palo Alto, CA. His research interests include 2D/3D computer vision, pattern recognition, and image processing. 


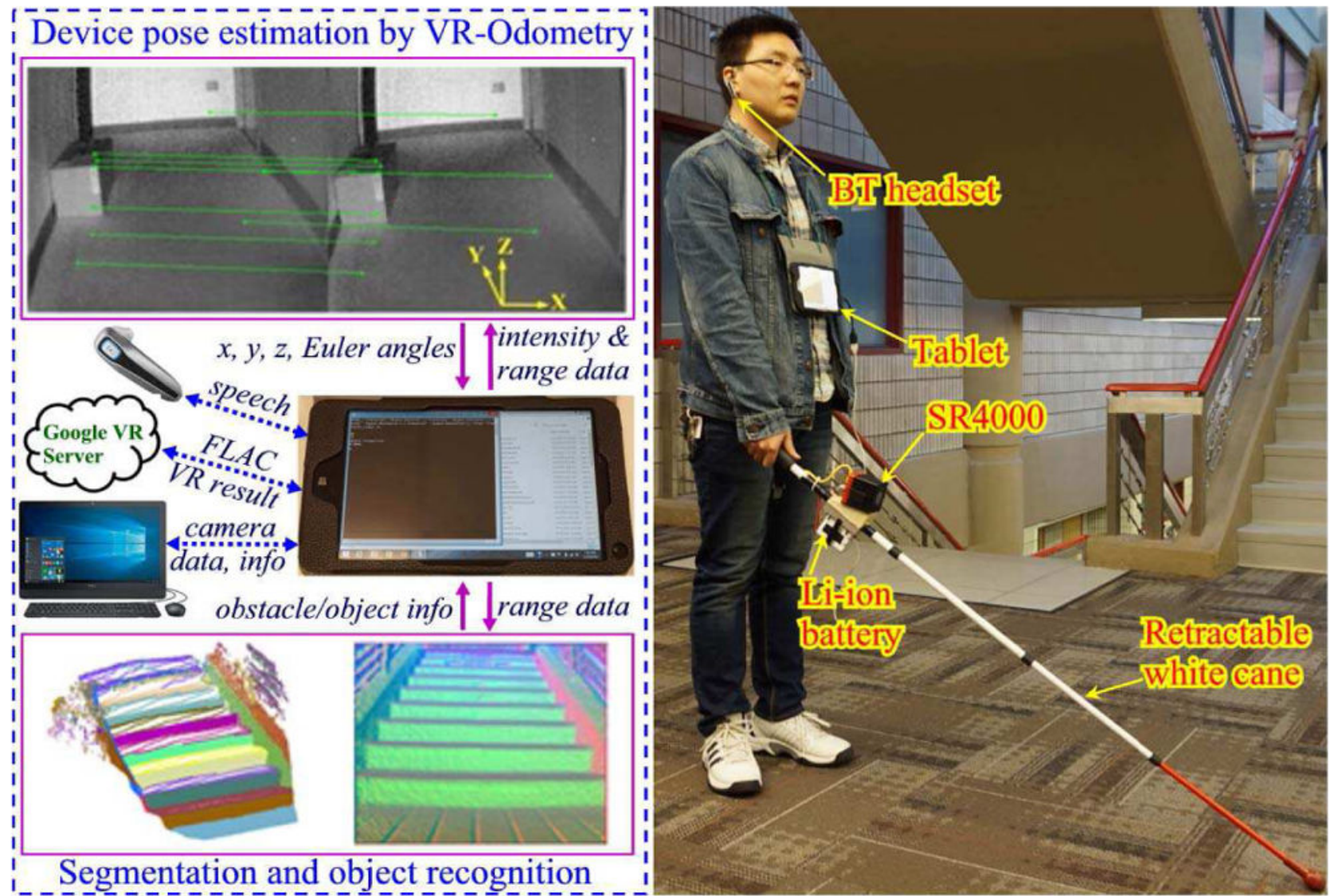

Fig. 1.

The Smart Cane: a computer-vision-enhanced white cane 


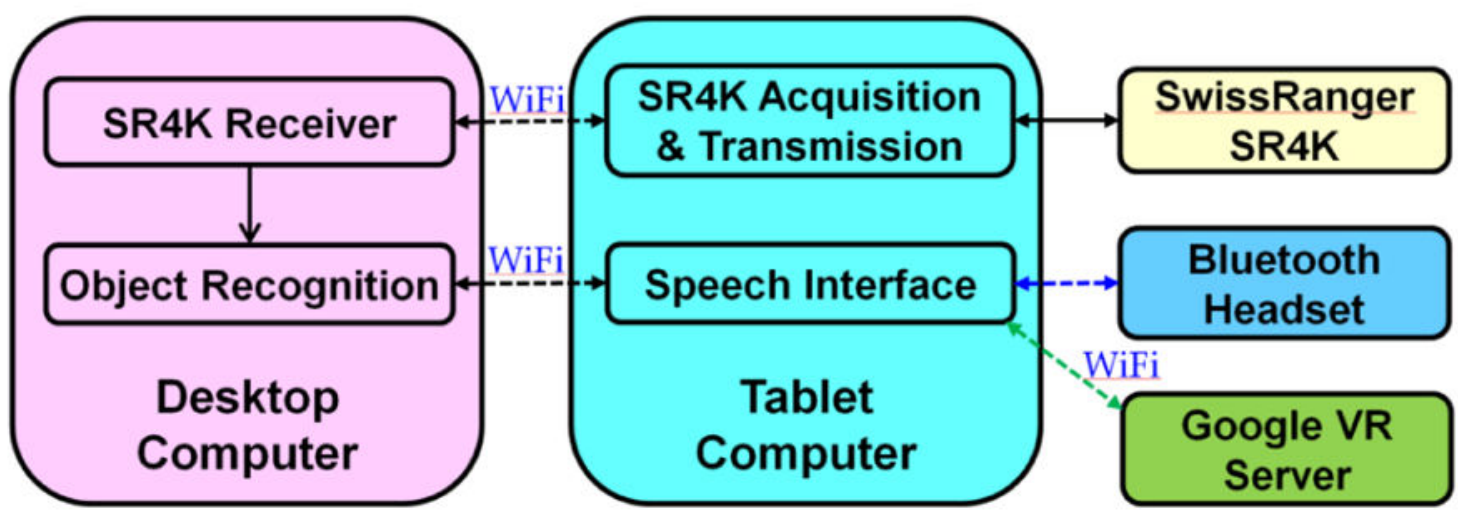

Fig. 2.

Diagram of the object recognition software of the SC prototype 


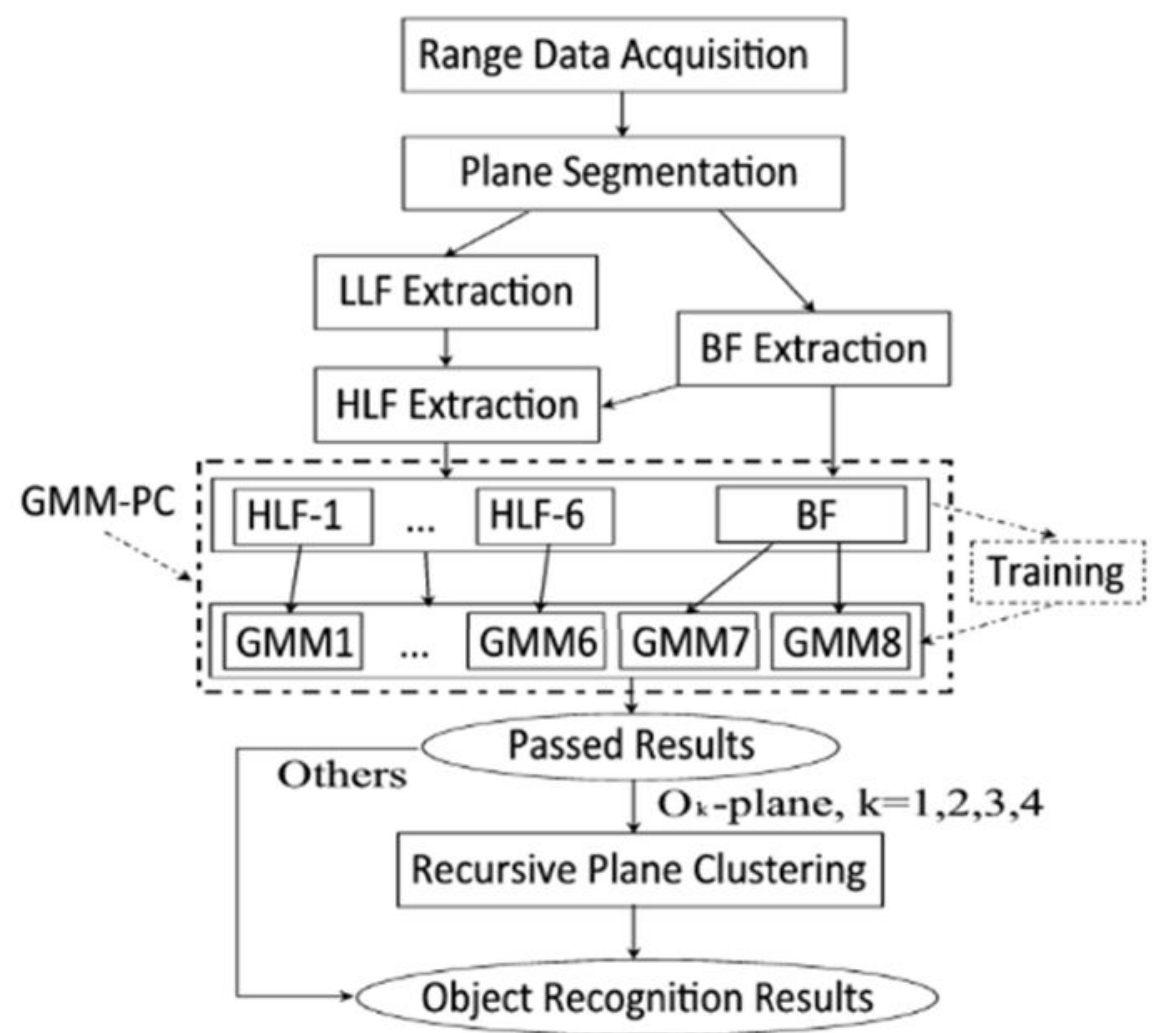

Fig. 3.

Diagram of the proposed object recognition method 

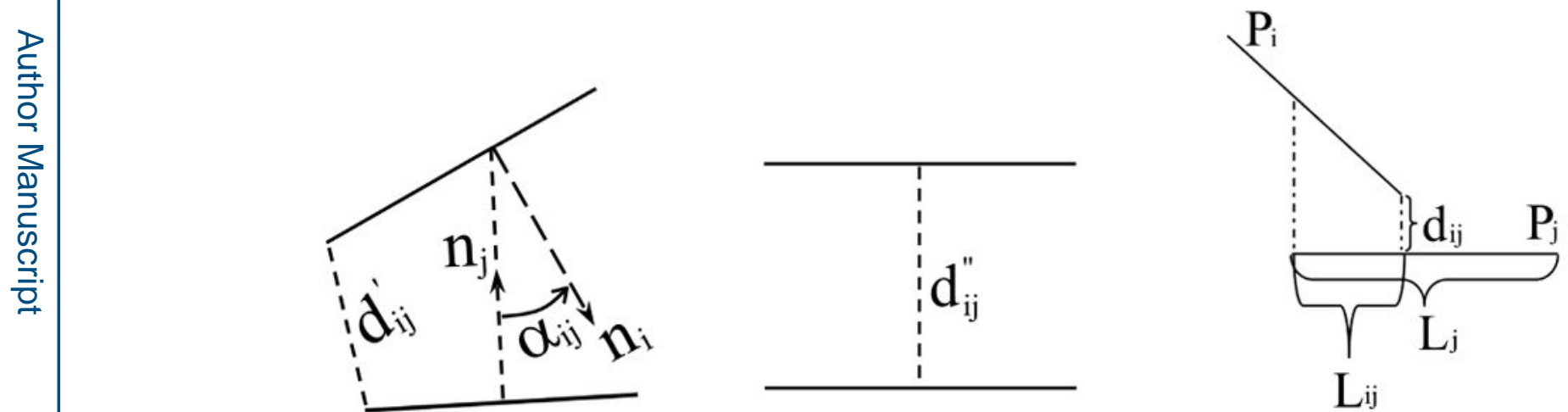

Fig. 4.

Definition of LLFs: (a) plane-distance $\left(d_{i j}^{\prime}\right)$ and plane-angle $\left(a_{i j}\right)$; (b) parallel-distance $\left(d_{i j}^{\prime \prime}\right)$; (c) projection-overlap-rate and projection-distance 


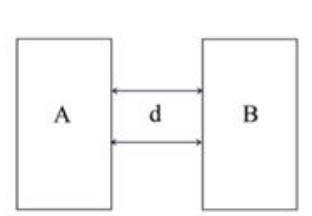

(a)

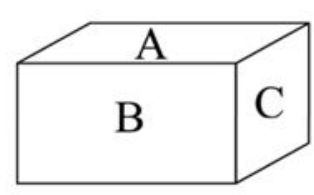

(d)

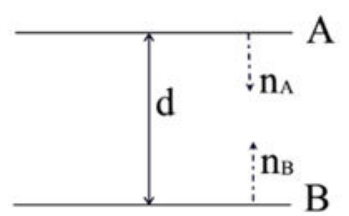

(b)

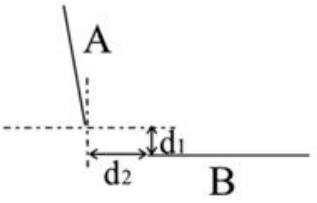

(e)

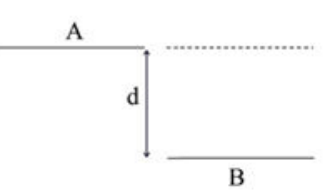

(c)

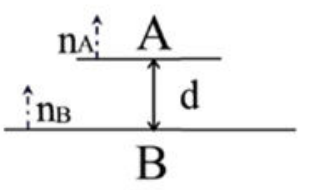

(f)

Fig. 5.

Definition of HLFs: (a) HLF-1; (b) HLF-2; (c) HLF-3; (d) HLF-4; (e) HLF-5: $d_{1}$ is $A$ 's projection-distance while $d_{2}$ is the minimum distance between $B$ 's points and $A$ 's projectionpoints on $B$; (f) HLF-6. For simplicity, we use $A, B$, and $C$ to represent any three patches $P_{i}$, $P_{j}$, and $P_{k}$ out of the $N$ planar patches. 


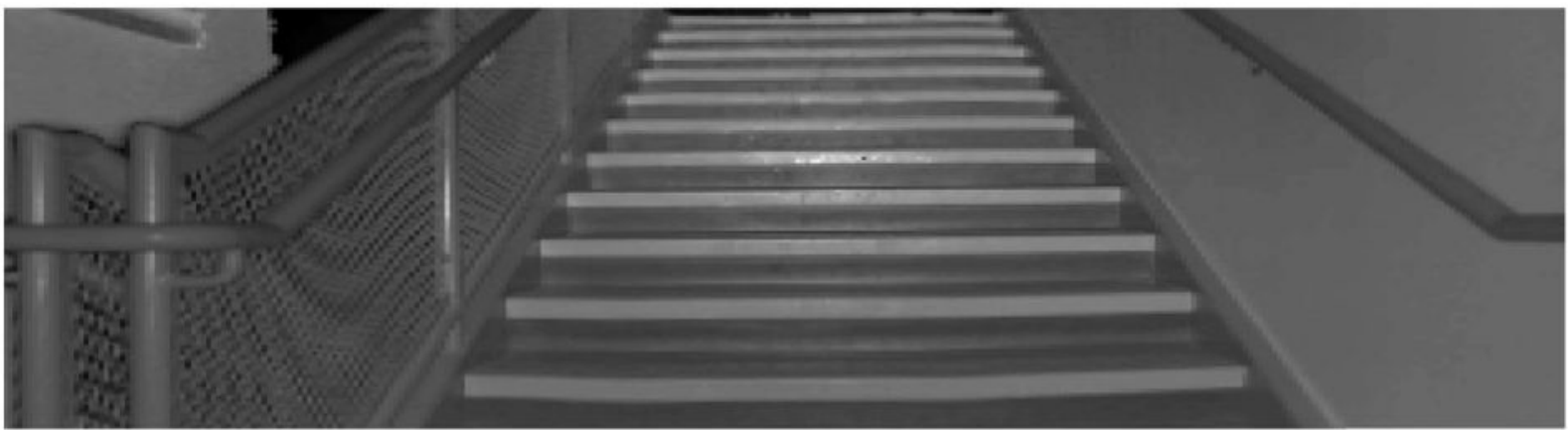

(a)

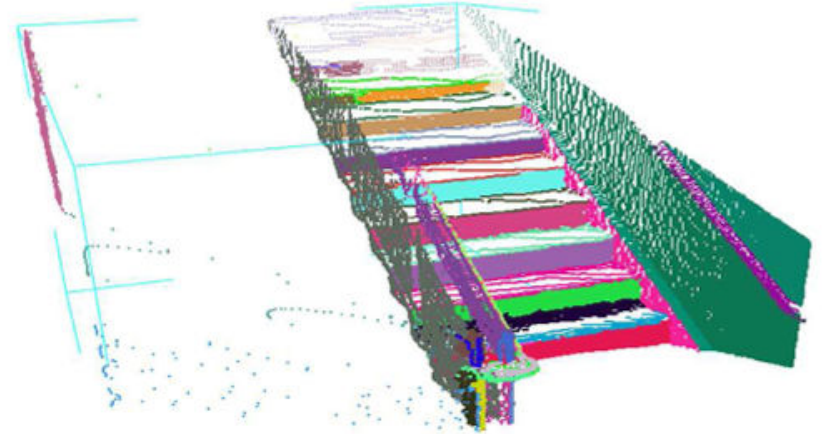

(b)

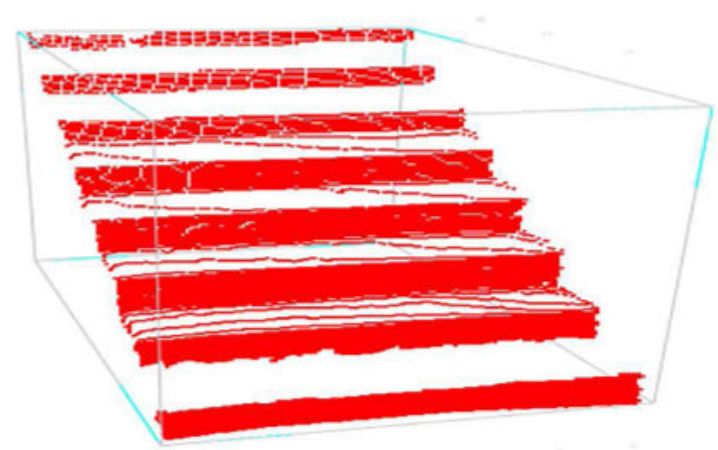

(c)

Fig. 6.

Experimental result with a stairway scene (multi-view data): (a) intensity image; (b) 3D view of the extracted planes; (c) 3D view of the detected stairway (labeled in red). 


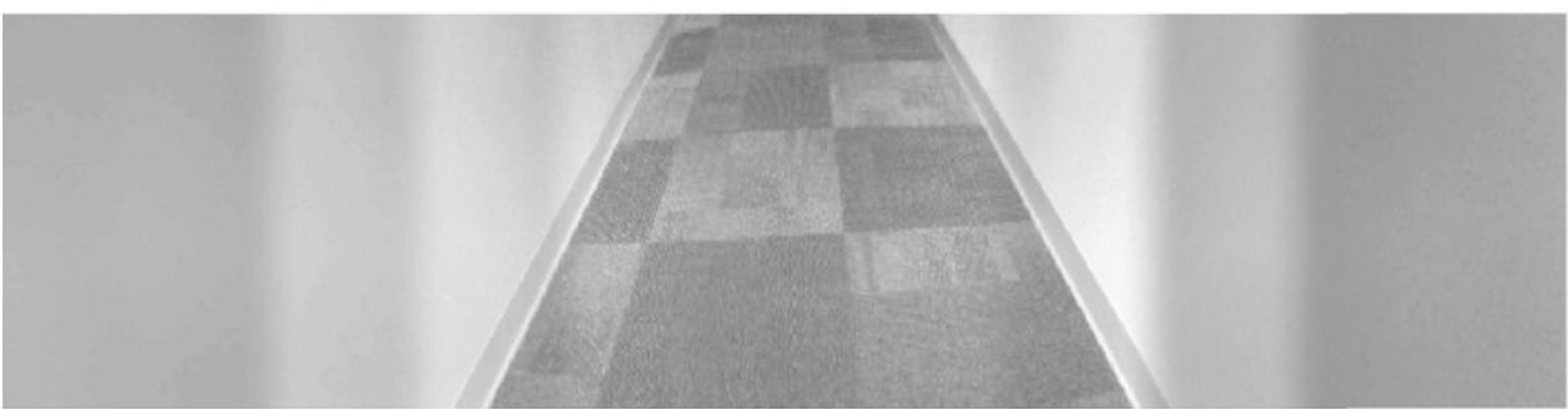

(a)

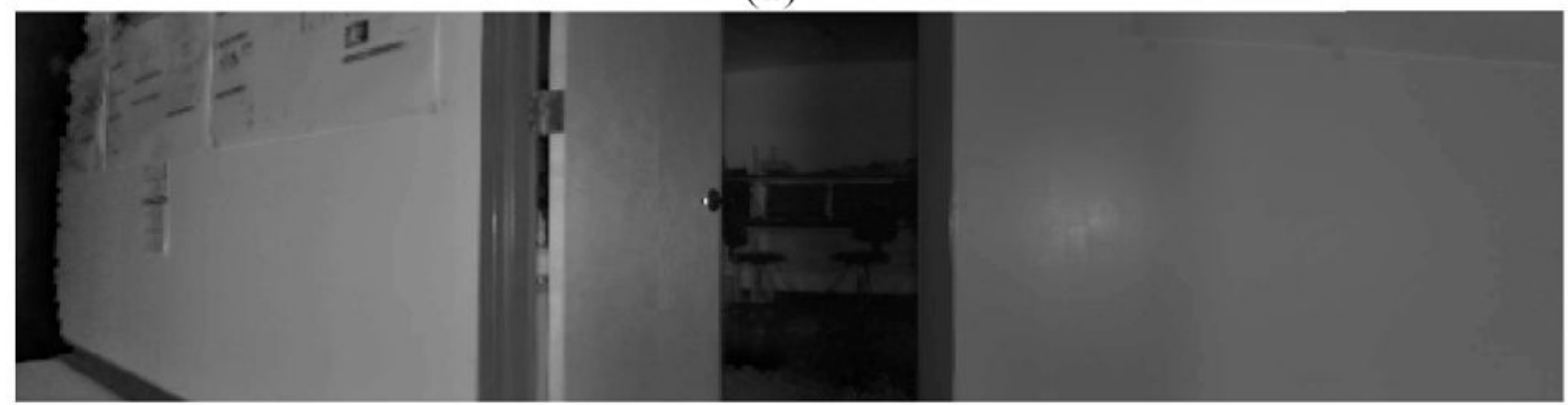

(b)

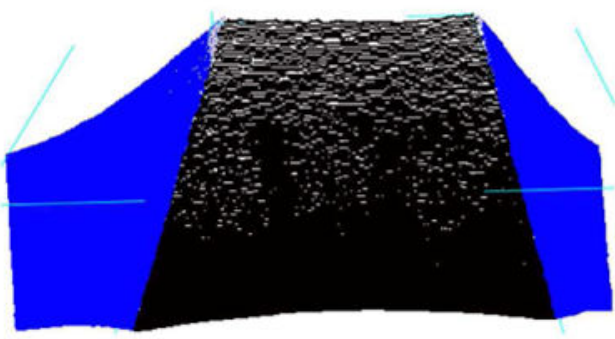

(c)

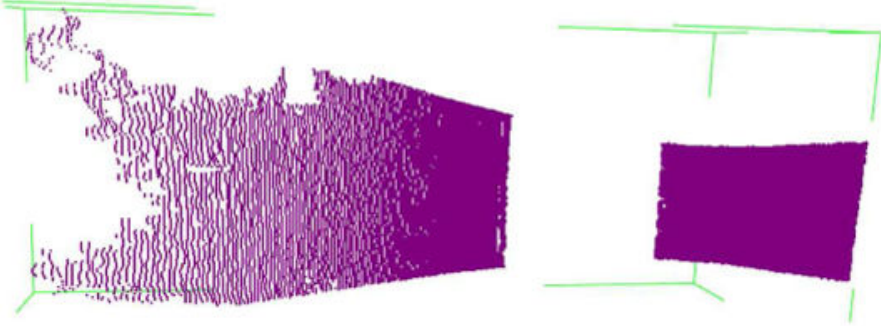

(d)

Fig. 7.

Experimental result with a hallway and a doorway scenes (multiview data): (a)/(b) intensity image; (c)/(d) 3D view of the detected hallway/doorway: hallway/doorway is labeled in blue/purple and ground in black. 


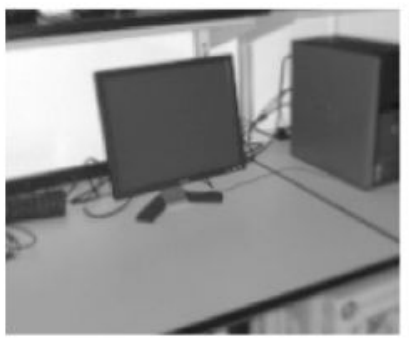

(a)

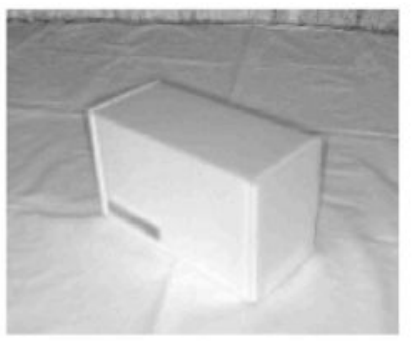

(b)

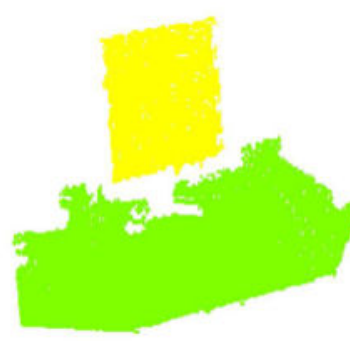

(c)

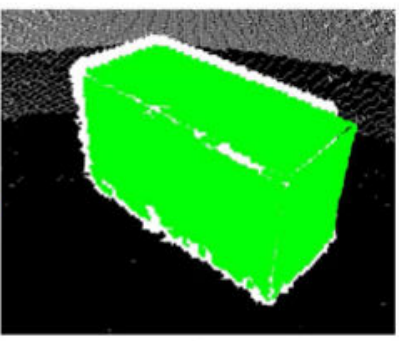

(d)

Fig. 8.

Experiment results with a monitor and a box scenes (single view data): (a)/(b) intensity image; (c)/(d) 3D view of the detected object. Monitor is (yellow); Table (light green); Parallelepiped (green); Ground (black). 


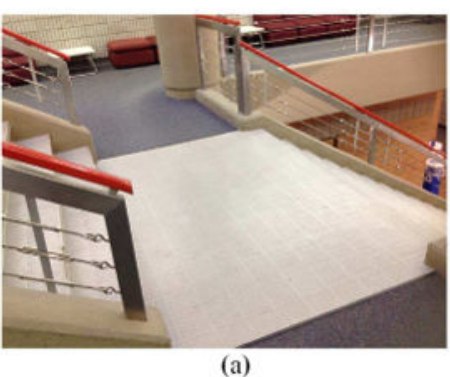

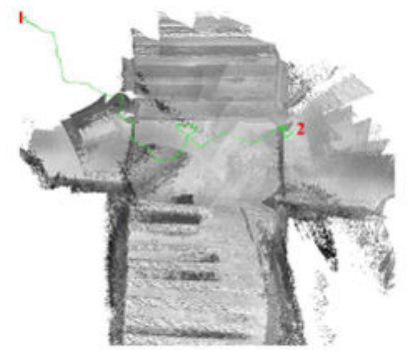

(b)

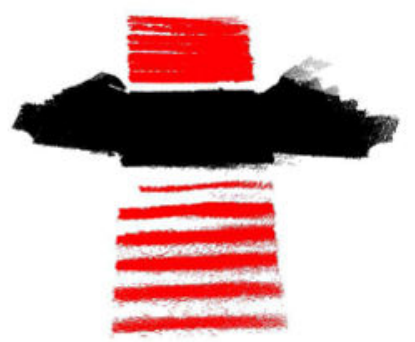

(c)

Fig. 9.

Experimental results with a stairway scene: (a) snapshot of scene; (b) 3D point cloud of multi-view data registered by using the SLAM method (green curve shows the SC's trajectory); (c) 3D view of the detected objects: ground (black), wall (blue) and stairways (red). The SC user walked from 1 to 2. 


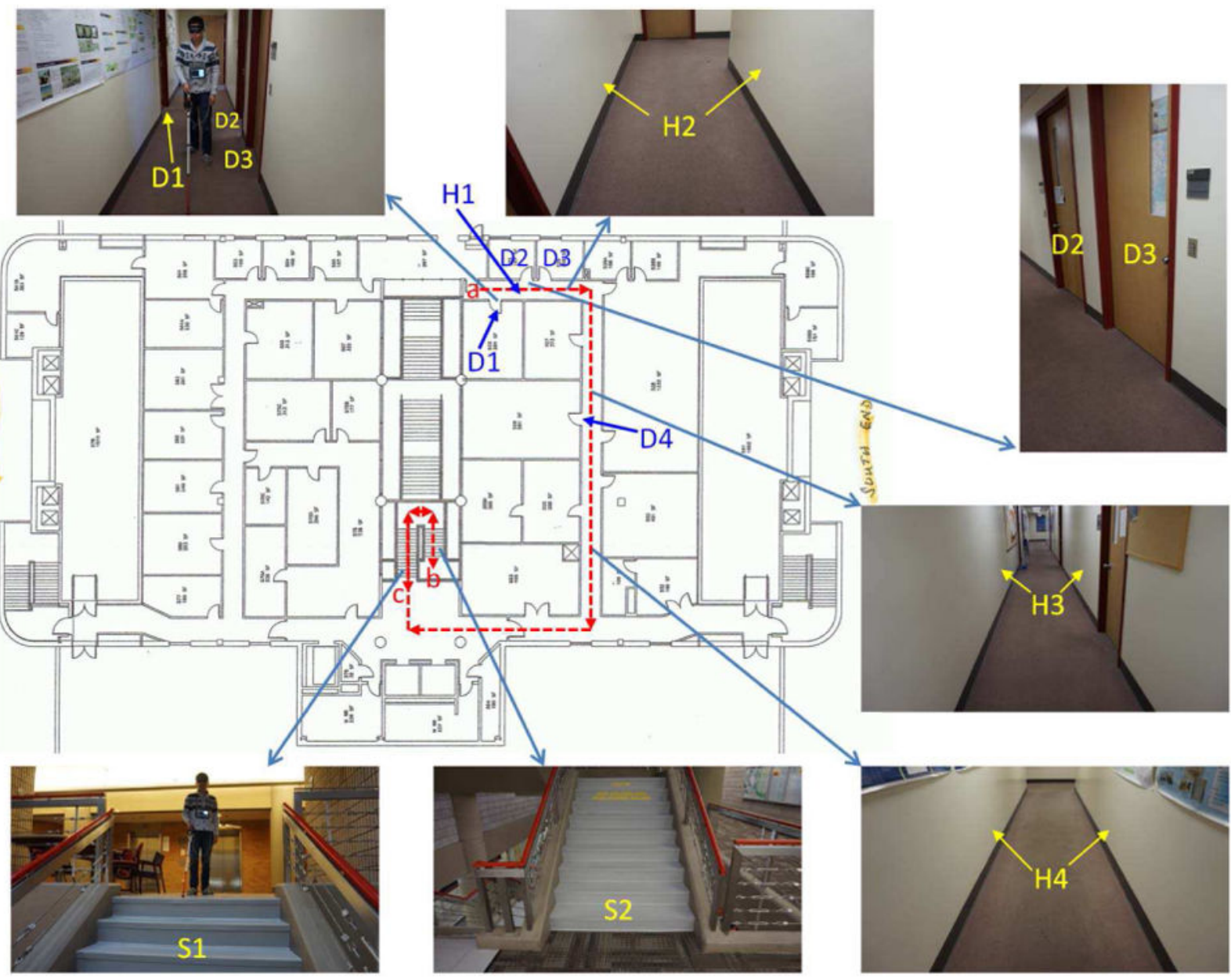

Fig. 10.

Map of $5^{\text {th }}$ floor in ETAS building for human subject test 


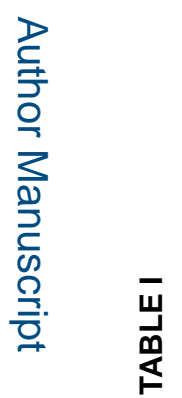

로을

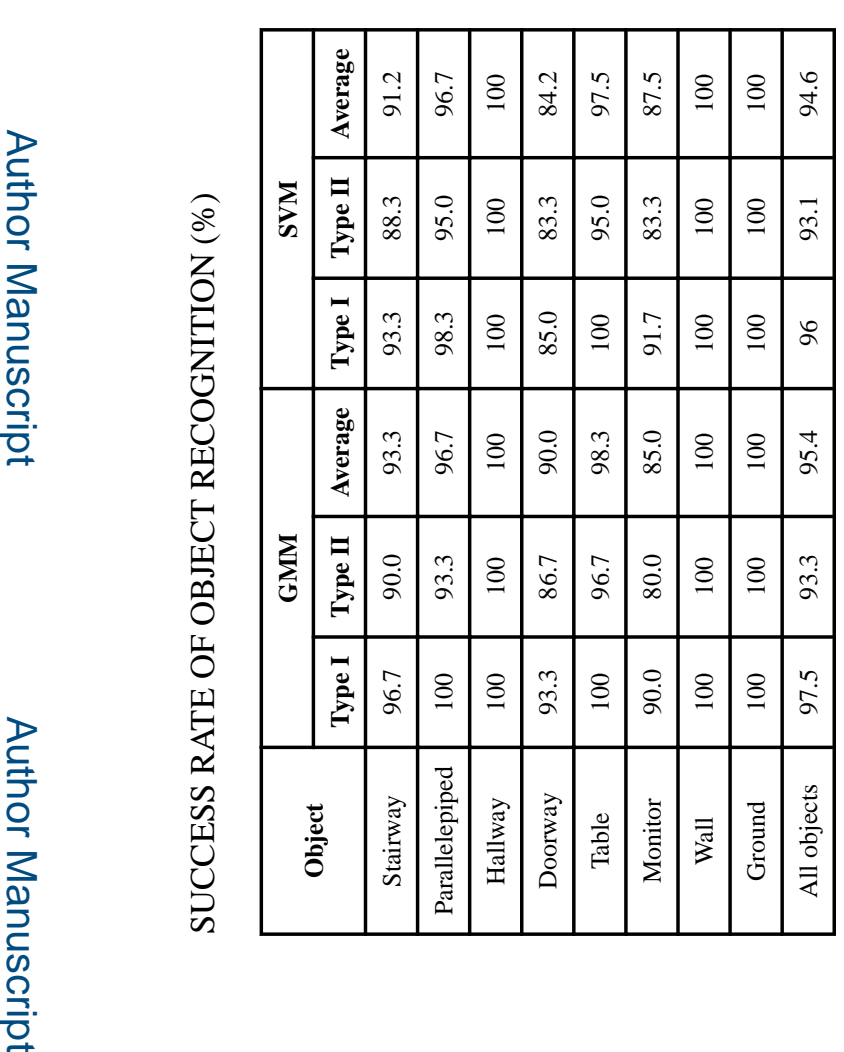

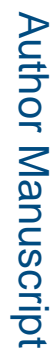

IEEE Trans Neural Syst Rehabil Eng. Author manuscript; available in PMC 2019 February 01. 


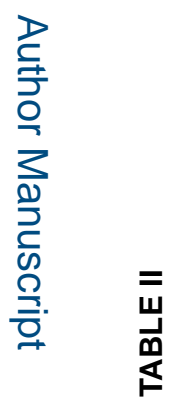

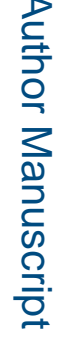

\begin{tabular}{|c|c|c|c|c|c|c|c|c|c|c|}
\hline \multirow{3}{*}{$\sum_{\infty}$} & 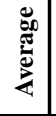 & $\stackrel{\overbrace{}}{\sigma}$ & 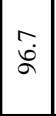 & \& & $\begin{array}{l}\text { I } \\
\mathbb{\infty}\end{array}$ & $\begin{array}{l}n \\
\vdots \\
a\end{array}$ & 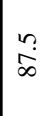 & 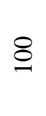 & $\nsubseteq$ & $\begin{array}{l}0 \\
\text { di }\end{array}$ \\
\hline & 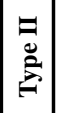 & $\begin{array}{l}m \\
\infty \\
\infty \\
\infty\end{array}$ & $\begin{array}{l}\dot{a} \\
\ddot{a}\end{array}$ & \& & $\underset{\infty}{m}$ & $\begin{array}{l}\ddot{2} \\
\dot{\alpha}\end{array}$ & $\underset{\infty}{\infty}$ & $\nsubseteq$ & $\nsubseteq$ & $\bar{\sigma}$ \\
\hline & 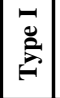 & $\stackrel{m}{\kappa}$ & $\begin{array}{c}m \\
\infty \\
\infty\end{array}$ & $\nsubseteq$ & $\begin{array}{l}0 \\
\dot{\infty} \\
\dot{\infty}\end{array}$ & $\nsubseteq$ & $\stackrel{i}{a}$ & $\stackrel{8}{ }$ & $\nsubseteq$ & \& \\
\hline \multirow{3}{*}{$\sum_{j=}$} & 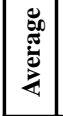 & $\stackrel{m}{\alpha}$ & $\hat{\sigma}$ & $\nsubseteq$ & $\stackrel{\circ}{\circ}$ & $\begin{array}{l}m \\
\infty \\
\infty\end{array}$ & $\underset{\dot{\infty}}{\stackrel{\circ}{0}}$ & $\nsubseteq$ & 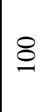 & $\stackrel{\dot{\leftrightarrow}}{\leftrightarrow}$ \\
\hline & 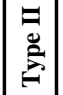 & $\stackrel{\dot{Q}}{\dot{\alpha}}$ & ๙ু & $\nsubseteq$ & $\stackrel{\circ}{\circ}$ & $\grave{\delta}$ & $\stackrel{\circ}{\dot{\infty}}$ & $\nsubseteq$ & $\stackrel{8}{\circ}$ & $\tilde{\sigma}$ \\
\hline & 离 & $\hat{\circ}$ & 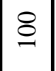 & 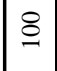 & m. & 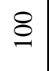 & 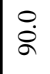 & $\nsubseteq$ & 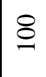 & $\begin{array}{l}n \\
\vdots \\
a\end{array}$ \\
\hline & 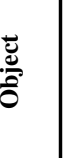 & 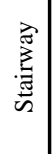 & 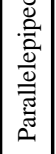 & 宽 & $\begin{array}{l}\text { 密 } \\
\stackrel{0}{0} \\
\stackrel{0}{0}\end{array}$ & 焉 & 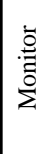 & 產 & 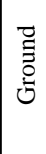 & \\
\hline
\end{tabular}

IEEE Trans Neural Syst Rehabil Eng. Author manuscript; available in PMC 2019 February 01. 